\title{
Route Duration in Mobile Ad hoc Networks
}

\author{
Michael Pascoe*, Javier Gomez, Victor Rangel \\ Department of Electrical Engineering \\ National University of Mexico, Mexico City, Mexico \\ Email: \{michael,javierg,victor\}@fi-b.unam.mx
}

\author{
Miguel Lopez-Guerrero \\ Department of Electrical Engineering \\ Metropolitan Autonomous University, Mexico City, Mexico \\ milo@xanum.uam.mx
}

\begin{abstract}
This paper presents an analytical and simulationbased work which helps to estimate time duration of routes in a mobile ad-hoc network. First, we studied a simple route with a single intermediate node between source and destination, and then we model how long it takes for the intermediate node to leave the route (i.e., leave the overlapping region formed by the intersection of the coverage areas between source and destination). Then, we generalized the model for routes with $\mathbf{N}$ intermediate nodes. Simulation experiments were developed using the NS-2 network simulator to verify that our theoretical model agrees with simulation results.
\end{abstract}

Keywords: ad hoc networks, duration of routes.

\section{INTRODUCTION}

An ad hoc network is a collection of wireless nodes dynamically forming a temporary network without use of any existing network infrastructure or centralized administration. Due to the limited transmission range of wireless network interfaces, multiple network interlinks or "hops" may be needed for one node to exchange data with another node across the wireless network. Network nodes are free to move randomly and organize arbitrarily; thus, the network's wireless topology may change rapidly and unpredictably. Such a network may operate in a standalone fashion, or may be connected to the larger Internet.

In general, there will be a need for none, one, or several intermediate forwarding nodes between source-destination pairs, depending on the separation distance between source and destination nodes. Figure 1a illustrates a route from a source node $S$ to a destination node $D$ involving several forwarding nodes. Each circle in Figure 1a represents the transmission range of each node in this route.

This paper presents analytical and simulation-based work which helps to predict the time duration of routes in a mobile ad-hoc network. Previous researchers have approached a similar problem, but they have relied on simulation and/or empirical results. We believe an analytical study is more powerful and has a higher applicability because it is not tied to any specific simulation scenario. The rest of the paper is as follows: Section II presents a brief description of previous work in this area. Section III presents a study of route duration in mobile ad hoc networks, we analyze a simpler 3-node routes first, and then we generalize the model to routes with $N$ intermediate nodes. Finally, in Section IV we present some final remarks.

\footnotetext{
*Supported by a UNAM/DGEP scholarship.
}

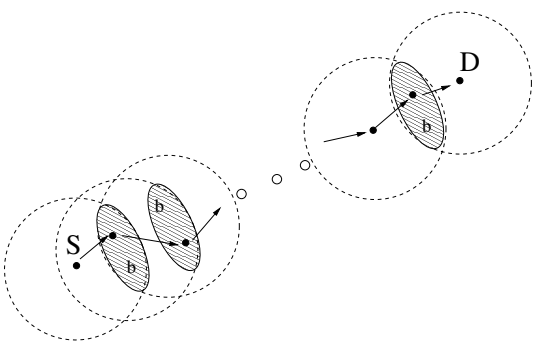

(a) Multihop Routing

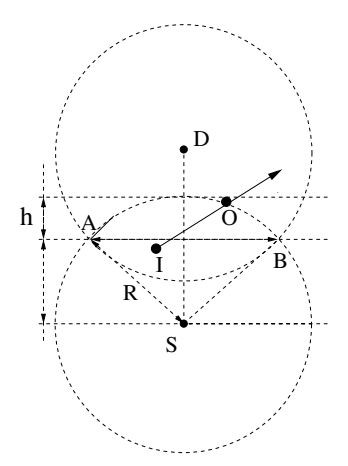

(b) Overlapping Region

Fig. 1. Multihop routing: (a) Example of a route involving several intermediate hops, (b) Overlapping region between two forwarding hops

\section{RELATED WORK}

Simulation has been the main method for analyzing the properties of route or path duration in mobile ad hoc networks in the past. Bai et al. [4] made one of the first studies concerning the analysis of path duration. Based on experimental results obtained by simulations, they assume that the lifetime of a path with four or more hops can be approximated by an exponential distribution. To cope with this limitation, Han et al. [5] use the Palmś theorem to state that, under some circumstances, the lifetime associated to paths with a large number of hops converges to an exponential distribution [5]. The popularity of the exponential fitting has made a common approximation in some other works as in [7] [8].

Other authors have analyzed path duration by means of empirical results. For instance, in [9] the authors have shown that the mean residual lifetime of routes depends on the number of hops, as well as on the mean link duration. On 
the other hand, in [3] the authors analytically prove that the average lifetime of a path decreases with its length. An analytical study on this issue is due to Tseng et al. [10]. In [11], the authors formally describe the distribution function of path duration assuming that nodes move according to a constant velocity model.

The model proposed by [2] uses a fluid flow mobility model to compute the crossing rate across the overlapping region. This model assumes that each forwarding node is found right after it enters the overlapping region, but it does not consider the possibility that the forwarding node is already located within the overlapping region, which is the case in a real scenario.

\section{Route Permanence Analysis}

The following theoretical analysis tries to solve most of the limitations found in [2]. We approach this complex problem by modeling and understanding simpler scenarios first, before moving to more complex scenarios. First, a route formed by three nodes will be analyzed considering that only the intermediate forwarding node is mobile while source and destination nodes remain static. Then, a route formed by three mobile nodes will be analyzed. Finally, a general case of a route formed by $N$ intermediate nodes will be analyzed.

\section{A. $S$ and $D$ are static}

In [2] it is assumed that a forwarding node is found right after it enters the overlapping region (see Figure 1b), and thus the time duration the node remains within this region is the longest. In a real scenario, forwarding nodes would already be found inside the overlapping region, and thus the time duration within the overlapping region would be shorter than in [2]. To include this situation into our analysis, it is necessary to consider all the possible initial positions and trajectories of the forwarding node within the overlapping region. Once this is done, the time interval the forwarding node remained inside the overlapping region can be found if the speed of movement is known.

Now let the source and destination nodes are placed at points $S\left(x_{S}, y_{S}\right)$ and $D\left(x_{D}, y_{D}\right)$, respectively (see Figure $1 \mathrm{~b}$ ), and let their transmission range be $R$ meters, so the covering zone of each node has the shape of a circle with radius $R$. Factor $h$ in Figure 1b plays a crucial role in the operation and performance of routing protocols for wireless ad hoc networks because it represents the size of the overlapping region (i.e., oval). As illustrated in Figure $1 \mathrm{~b}, h$ accounts for how much area overlaps between adjacent forwarding nodes. Furthermore, let points $A\left(x_{A}, y_{A}\right)$ and $B\left(x_{B}, y_{B}\right)$ be the intersection points between both circles, these points can be found by:

$$
\begin{aligned}
x_{A / B} & =\frac{-B \prime \pm \sqrt{B \prime^{2}-4 A \prime C \prime}}{2 A \prime} \\
y_{A / B} & =\frac{2\left(x_{D}-x_{S}\right) x_{A / B}+\left(x_{S}^{2}-x_{D}^{2}\right)+\left(y_{S}^{2}-y_{D}^{2}\right)}{2\left(y_{S}-y_{D}\right)}
\end{aligned}
$$

where:

$$
\begin{aligned}
& A^{\prime}=4\left[\left(x_{S}-x_{D}\right)^{2}+\left(y_{S}-y_{D}\right)^{2}\right] \\
& B^{\prime}=-4\left[\left(x_{S}^{2}-x_{D}^{2}\right)\left(x_{S}-x_{D}\right)+\left(y_{S}-y_{D}\right)^{2}\left(x_{S}+x_{D}\right)\right] \\
& C^{\prime}=\left[\left(x_{S}^{2}-x_{D}^{2}\right)-\left(y_{S}-y_{D}\right)^{2}\right]^{2}+4\left[\left(x_{S}^{2}-R^{2}\right)\left(y_{S}-y_{D}\right)^{2}\right]
\end{aligned}
$$

Let point $I\left(x_{i}, y_{i}\right)$ be the position of the intermediate node at time zero and point $O\left(x_{o}, y_{o}\right)$ be the position where the node leaves the overlapping region following a straight line trajectory sloped $\alpha$ degrees, moving at constant speed $v$. Then, the distance between the initial and final position of the intermediate node can be found by:

$$
d_{I-O}(\alpha)=a b s\left(-\sqrt{a^{2}+b^{2}} \sin (\alpha+\delta)+\cdots\right.
$$

$$
\left.\cdots+\sqrt{R^{2}-\left[\left(a^{2}+b^{2}\right) \cos ^{2}(\alpha+\delta)\right]}\right)
$$

where:

$$
a=\left(y_{i}-y_{S / D}\right) \quad ; \quad b=\left(x_{i}-x_{S / D}\right) \quad ; \quad \delta=\arctan \left(\frac{b}{a}\right)
$$

Then, average distance for a forwarding node given its initial position can be found by:

$$
\bar{d}_{I-O}=\frac{1}{\Delta \alpha} \int_{\alpha_{m}}^{\alpha_{M}} d_{I-O}(\alpha) d \alpha
$$

where:

$$
\begin{gathered}
\alpha_{m} \leq \alpha \leq \alpha_{M} \quad ; \quad \Delta \alpha=\left|\alpha_{M}-\alpha_{m}\right| \\
\alpha_{M}=\arctan \left(\frac{y_{B}-y_{i}}{x_{B}-x_{i}}\right) \quad ; \quad \alpha_{m}=\arctan \left(\frac{y_{A}-y_{i}}{x_{A}-x_{i}}\right) \\
\bar{d}_{I-O}=\frac{1}{\Delta \alpha}\left[\left.\sqrt{a^{2}+b^{2}} \cos (\alpha+\delta)\right|_{\alpha_{m}} ^{\alpha_{M}}+\cdots\right. \\
\left.\cdots+\int_{\alpha_{m}}^{\alpha_{M}} \sqrt{R^{2}-\left[\left(a^{2}+b^{2}\right) \cos ^{2}(\alpha+\delta)\right]} d \alpha\right]
\end{gathered}
$$

Integral $\int_{\alpha_{m}}^{\alpha_{M}} \sqrt{R^{2}-\left[\left(a^{2}+b^{2}\right) \cos ^{2}(\alpha+\delta)\right]} d \alpha$ shown as part of Equation 4 can not be solved exactly, but an approximated solution can be found replacing the square root by a binomial series and after some mathematical manipulation it can be simplified by:

$$
\sqrt{R^{2}-\left[\left(a^{2}+b^{2}\right) \cos ^{2}(\alpha+\delta)\right]}=R\left(1-k_{i}(v(\alpha))^{i}\right)
$$

where:

$v(\alpha)=\cos ^{2}(\alpha+\delta) \quad ; \quad k_{i}=\sum_{i=1}^{n} \frac{\prod_{i=1}^{n}\left(\frac{3}{2}-i\right)}{(i) !}\left(\frac{\left(a^{2}+b^{2}\right)}{R^{2}}\right)^{i}$

then:

$R \int(1-v(\alpha))^{1 / 2} d \alpha=R\left(\alpha-k_{i} \int \cos ^{2 i}(\alpha+\delta) d \alpha\right)$

So, average distance approximation $\left(\widetilde{d}_{I-O}\right)$ will be:

$$
\bar{d}_{I-O}=\frac{1}{\Delta \alpha}\left[\left.\sqrt{a^{2}+b^{2}} \cos (\alpha+\delta)\right|_{\alpha_{m}} ^{\alpha_{M}}+\cdots\right.
$$




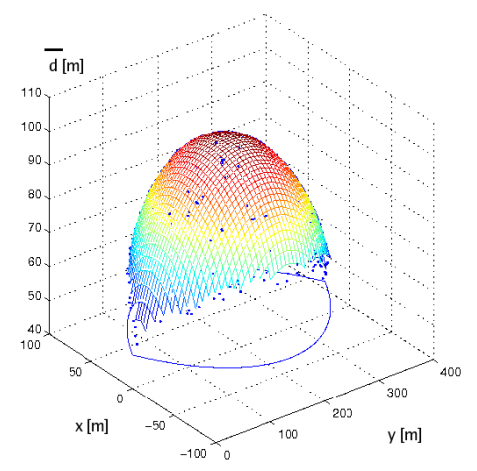

Fig. 2. Average distance distribution for several forwarding nodes

$$
\cdots+R\left(\alpha-k_{i} \int_{\alpha_{m}}^{\alpha_{M}} \cos ^{2 i}(\alpha+\delta) d \alpha\right)
$$

The average distance for a forwarding node on a random initial position $\left(x_{i}, y_{i}\right)$ could be found by:

$$
\bar{d}_{I-O}=\frac{1}{\Delta \alpha} \frac{1}{\Delta y_{i}} \frac{1}{\Delta x_{i}} \int_{\alpha_{m}}^{\alpha_{M}} \int_{y_{i_{m}}}^{y_{i} M} \int_{x_{i_{m}}}^{x_{i}} d_{I-O}\left(x_{i}, y_{i}, \alpha\right) d x_{i} d y_{i} d \alpha
$$

where:

$$
\begin{gathered}
\alpha_{m} \leq \alpha \leq \alpha_{M} \quad ; \quad \Delta \alpha=\left|\alpha_{M}-\alpha_{m}\right| \\
x_{i_{m}} \leq x_{i} \leq x_{i_{M}} \quad ; \quad \Delta x=\left|x_{i_{M}}-x_{i_{m}}\right| \\
y_{i_{m}} \leq y_{i} \leq y_{i_{M}} \quad ; \quad \Delta y=\left|y_{i_{M}}-y_{i_{m}}\right|
\end{gathered}
$$

The average time for a forwarding node to remain inside the overlapping region $\left(T_{p}\right)$ when it is moving at a constant speed $(v)$, could be found by:

$$
T_{p}=\frac{\bar{d}_{I-O}}{v}
$$

The average distance and standard deviation can be calculated for several intermediate forwarding nodes randomly placed in the overlapping region, this is shown in Figure 2 and Figure 3, respectively. In these figures, it is clear that the values of average distance and standard deviation depend on the initial position where the forwarding node is located. The closer the forwarding node is to the border of the overlapping region, the lower the average distance (i.e., a shorter route duration), and the higher its standard deviation. This behavior can be explained because the forwarding node will experience either very short times (i.e., it crosses the border right away) or long times (i.e., it crosses the entire overlapping region before crossing the border) when it is near the boundary of the overlapping region.

\section{B. 3-node-mobile case}

In this scenario we analyze routes where source, destination and forwarding nodes are all mobile. Again we model how much time it takes for the intermediate node to exit the overlapping region. We consider all nodes are moving at a constant speed of $v=1 \mathrm{~m} / \mathrm{s}$ in this scenario with each node following a random trajectory. Results found for $v=1 \mathrm{~m} / \mathrm{s}$ can be extrapolated to other velocities by simply scaling (dividing) the results found by the desired velocity. This scenario is far

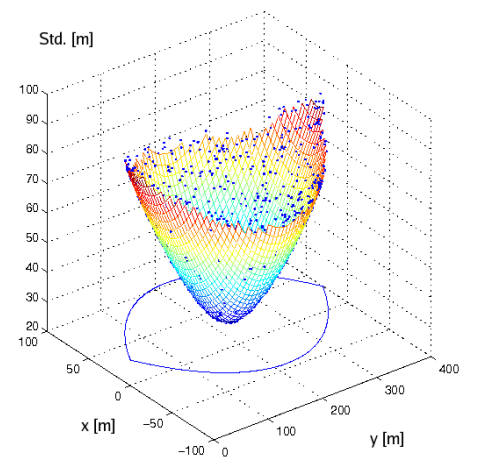

Fig. 3. Standard deviation distribution for several forwarding nodes

more complex to analyze than the simpler scenario because the size and location of the overlapping region (i.e., oval) is constantly changing as time passes. Given this complexity, we could not model this scenario analytically, as in the previous case, but we had to rely on a statistical model instead.

A statistical analysis of this case was achieved using data from different numerical experiments, in order to obtain a histogram showing the relative frequency of the time that each forwarding node remains inside the overlapping region of a certain route. The experiments run were as follows. At time zero, we positioned source and destination nodes to a fixed location so the size of the overlapping region (described by the $h$ parameter) was always constant for each experiment, then a node within the overlapping region was randomly selected as forwarding node between source and destination. We then assigned different trajectories to the three nodes involved and let the nodes move at a constant speed of 1 $\mathrm{m} / \mathrm{s}$. The experiment ended once the distance between either source-forwarding or forwarding-destination exceeded $R$ (the transmission range) which in this experiments corresponds to $250 \mathrm{~m}$, the transmission range of outdoor 802.11 radios. Again this analysis can be linearly scaled to other value of $R$.

Figure 4 show this histogram for $h=70 \mathrm{~m}$. This histogram represents the experimental probability distribution function (pdf) associated to the time that each forwarding node remains inside of each overlapping zone of a certain route. This pdf could be represented by the following mathematical expression:

$$
P(t)=\alpha_{1} e^{-\left(\frac{t-\beta_{1}}{\delta_{1}}\right)^{2}}+\alpha_{2} e^{-\left(\frac{t-\beta_{2}}{\delta_{2}}\right)^{2}}
$$

where, parameters $\alpha_{i}, \beta_{i}, \delta_{i}$ could be found using a curve fitting method (see the solid curve in Figure 4). While this model considers all possible positions of the forwarding node and all possible trajectories, it does not consider all the possible values of $h$ at time zero.

\section{General case; N-node-mobile}

Finally, we can now analyze routes involving a set of $N$ intermediate nodes. Once again we consider routes where all nodes move at a constant speed with randomly selected trajectories. This scenario is even more complex because 


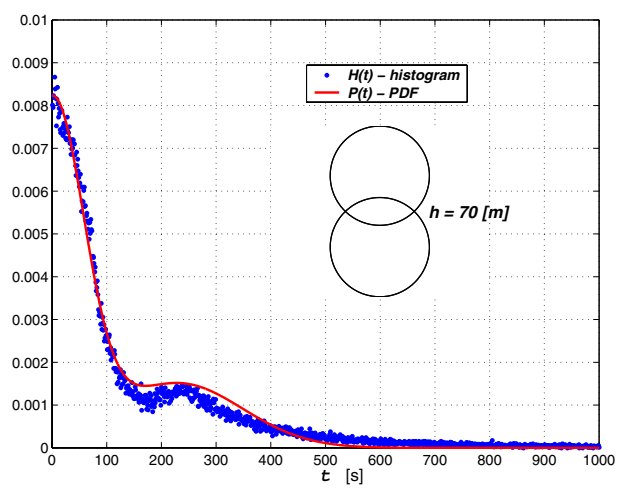

Fig. 4. PDF for $\mathrm{h}=70 \mathrm{~m}$

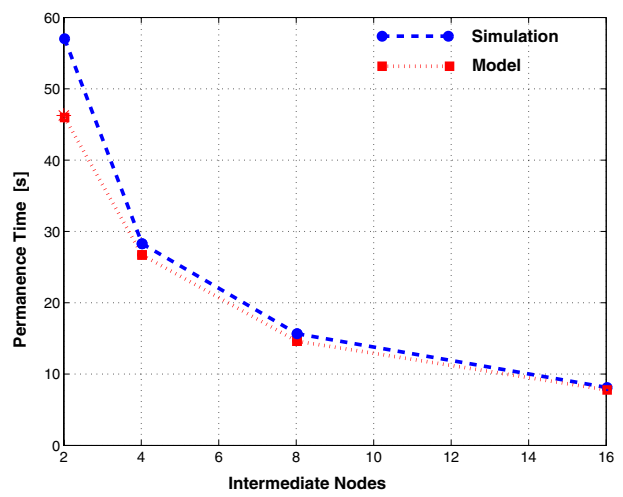

Fig. 5. Time duration of routes versus number of intermediate nodes

now several ovals, each with a different $h$, are involved. We approach this problem by breaking it into $N$ simpler 3node-mobile cases, which we already analyzed. The procedure we followed for each experiment was: first, we generated $N$ random variables $\left[T_{1}, T_{2}, \ldots, T_{N}\right]$ according to the pdf shown in Figure 4, with the outcome of each variable $T_{i}$ representing the time duration that each of the $N$ hops remained in the route. As we mentioned, each overlapping region for each of the $N$ hops will have a different size (a different $h$ value), however we chose a single pdf using an average overlapping region $(h=70 m)$. We can then compute the time duration of a route involving $N$ intermediate nodes, Troute $_{N}$, as

$$
\text { Troute }_{N}=\min \left[T_{1}, T_{2}, \ldots, T_{N}\right]
$$

We repeated these experiments hundreds of times for routes with $N$ hops and computed the average route duration in each case, results are shown in Figure 5. As expected, the duration of routes decreases as the number of intermediate nodes increases. We compared this results with simulations using the ns-2 network simulator [1]. The settings for the simulations consisted on a 2000 by 2000 meters network with 400 nodes. We used the random way-point mobility model with $v=1 \mathrm{~m} / \mathrm{s}$. We created routes in the simulator involving $N$ hops and then we let the simulation run until the first intermediate node left the route. We ran 100 experiments for each point and the results are also shown in Figure 5.
Relative errors between model and simulation results in Figure 5 were found around $20 \%$ for routes with 2 intermediate forwarding nodes, whereas relative errors oscillated between $6 \%$ and $3 \%$ for routes with 4,8 and 16 intermediate nodes. We consider the main reason why relative errors were larger for routes with small $N$ due to the variability of overlapping regions at time zero that we did not consider (we used a single value of $h$ in the model), whereas relative errors for routes with larger values of $N$ are smaller because the average value of $h$ for the $N$ hops is closer to the average value we used.

\section{CONCLUSIONS}

In this paper we propose a model to estimate duration of routes in wireless ad hoc networks when nodes move following random directions and moving at a constant speed. Theoretical, numerical and simulations experiments were developed. In general, simulation results are very close to the results obtained by using the proposed model. Results from this work can be used to compute the signaling overhead of unicast and multicast routing protocols for mobile ad hoc networks because every time a route is broken, the routing protocol needs to either repair the route locally or find a new route.

Acknowledgments: This work is supported in part by research funds from CONACyT grant 47197-Y and PAPIIT grant IN110805.

\section{REFERENCES}

[1] Kevin Fall, Kannan Varadhan, The NS Manual, The VINT project, UC Berkeley, LBL, USC/ISI and Xerox PARC, July 16, 2003.

[2] Javier Gomez and Andrew T. Campbell, Using Variable-Range Transmission Power control in Wireless Ad hoc Networks, IEEE Transactions on Mobile Computing, Vol. 6, No. 1, January 2007.

[3] D. Turgut and S.K. Das and M. Chatterjee, Longevity of Routes in Mobile Ad hoc Networks, Proc. of IEEE Vehicular Technology Conference VTC 2001, 2833-2837 vol.4, Rhodes Greece, Spring 2001.

[4] F. Bai and N. Sadagopan and B. Krishnamachari and A. Helmy, Modelling Path Duration Distributions in MANETS and Their Impact on Reactive Routing Protocols, IEEE J. on Selected Areas in Communications, v. 22, 1357- 1373, no. 7, pp. 1357- 1373, September 2004.

[5] Y. Han and R. La and A. Makowski and S. Lee, Distribution of Path Durations in Mobile Ad hoc Networks - Palm's Theorem to the Rescue. To appear in Computer Network.

[6] S. Jiang and D. He and J. Rao, A Prediction-based Link Availability Estimation for Mobile Ad Hoc Networks, IEEE/ACM Trans. on Networking, Issue 6, pp.1302 - 1312, December 2005.

[7] J. Brosch and D. A. Maltz and D. B. Johnson and Y.C. Hu and and J. Jetcheva, A Performance Comparison of Multi-Hop Wireless Ad Hoc Network Routing Protocols, Proc. of MOBICOM, Dallas (USA), Oct. 1998

[8] S. Arbindi and K. Namuduri and R. Pendse, Statistical Estimation of Route Expiry Times in On-demand Ad hoc Routing Protocols, Proc. of the Second IEEE International Conference on Mobile Ad Hoc and Sensor Systems (MASS 05), Washington (USA), November 2005.

[9] S. Cho and J.P. Hayes, Impact of Mobility on Connection Stability in Ad Hoc Networks Proc. of IEEE Communication Society, WCNC 2005, vol. 3, pp.1650-1656, New Orleans (USA).

[10] Y.C. Tseng and Y.F. Li and and Y.C. Chang, On Route Lifetime in Multihop Mobile Ad Hoc Networks, IEEE Trans. on Mobile Computing, Vol. 2, No. 4, pp. 366-376, October 2003.

[11] D. Yu and H. Li and I. Gruber, Path Availability in Ad Hoc Network, in Telecommunications, ICT 10th International Conf. vol.1, pp.383-387, March 2003. 